\title{
Which Disease and Individual-Based Factors Predict Intentions to Undergo Whole Genome Sequencing?
}

\author{
Felicity Kiln', Alana Fisher, ${ }^{1,2}$, Ilona Juraskova1,2 \\ ${ }^{1}$ School of Psychology, The University of Sydney, Sydney, Australia \\ ${ }^{2}$ Centre for Medical Psychology and Evidence-Based Decision-Making (CeMPED), The University of Sydney, \\ Sydney, Australia \\ Email: felicitykiln@gmail.com, alanacfisher@gmail.com, ilona.juraskova@sydney.edu.au
}

Received 14 August 2014; revised 12 September 2014; accepted 10 October 2014

Academic Editor: Kate Sweeny, University of Bergen, Norway

Copyright (C) 2014 by authors and Scientific Research Publishing Inc.

This work is licensed under the Creative Commons Attribution International License (CC BY). http://creativecommons.org/licenses/by/4.0/

(c) (i) Open Access

\begin{abstract}
Purpose: The past decade has seen rapid acceleration in the public's access to Whole Genome Sequencing (WGS) technology, however, factors that may influence a person's decision to undergo this complex health screening have received little empirical attention. This is the first psychosocial study to investigate which disease and individual-based factors predict intention to undergo WGS. Methods: A total of 164 first-year university students responded to hypothetical disease scenarios (varied by disease penetrance and treatment availability) and completed self-report measures of individual factors. Results: Intention to undergo WGS was significantly higher in the presence of available treatment and high disease penetrance $(p<0.05)$. There was also a significant interaction between treatment and disease penetrance on intention $(p<0.001)$. Task self-efficacy, positive outcome expectancy and attitude towards uncertainty all significantly predicted WGS intention $(p<0.05)$. Conclusions: Treatability and disease penetrance appear to be two distinct motivations that can also interact to influence intention to pursue WGS. Task self-efficacy, positive outcome expectancies and uncertainty avoidance are likely to motivate intention to pursue WGS in young healthy adults. These findings will be useful in informing the optimal design of WGS psycho-educational resources and screening provider protocols.
\end{abstract}

\section{Keywords}

Whole Genome Sequencing, Disease Penetrance, Treatment Availability, Psychological Factors, Theoretical Model 


\section{Introduction}

Rapid developments in clinical genomics over the past decade has revolutionised DNA analysis by enabling an individual to obtain their entire genome sequence in one test [1]. This new type of genetic health screening, called Whole Genome Sequencing (WGS), will allow healthy individuals to learn their vulnerability to numerous single and multi-gene disorders [2]. Knowledge of one's own genomic profile can provide warnings and the opportunity for preventative actions, specialised treatment and pharmacotherapy interventions for the individual [1]. However, given the immense scope and variety of genomic information, public access to this new technology presents numerous ethical and psychosocial challenges [3]. Considering the rapid pace at which WGS technology is advancing, coupled with its falling cost, there is an increasing need to understand the factors that motivate people to undertake WGS testing, and the psychological consequences of the test results.

Whilst there is a lack of psychosocial research exploring factors influencing intention to undergo WGS and the associated benefits and risks [4], studies in a genetic setting offer valuable information for guiding research in this novel area. A number of psychosocial genetic studies have identified penetrance, defined as the probability of getting ill when a person is a mutation carrier, as a key predictor of test-taking intentions. In a sample of healthy university students, intention to undergo testing for Alzheimer's Disease (AD) was significantly higher when a positive result denoted a $90 \%$ chance of developing the disease, compared to a 50\% chance [5]. In a more recent study, healthy adults indicated highest intention to pursue genetic testing when a positive result suggested an almost certain probability (90\%) they will develop a disease, compared to an uncertain probability (50\%) [6].

Apart from penetrance, the availability of treatment has also been found to influence intention to undertake genetic testing. In a study by Shaw and Bassi [7], healthy adults expressed greater intention to undergo genetic screening for curable diseases, than for those with no cure, which is consistent with people's desire to assert control in their lives. Similarly, Roberts [8] found that first-degree relatives of people with Alzheimer's were more likely to report test-taking intention when presented with hypothetical scenarios where treatment to prevent or delay disease onset was available. In a university sample there was greater interest in testing for an unspecified cancer than AD, likely due to the lack of treatments available for AD compared to many cancers [5]. Therefore, disease characteristics namely penetrance and treatment availability, may influence intention to pursue WGS among healthy young adults.

There is also strong evidence in the health psychology literature for the role of individual factors, including task self-efficacy, outcome expectancies and risk perception, in explaining cognitive and emotional influence on health behaviours, including genetic testing. Individuals expressing high task self-efficacy, or the perceived ability to successfully carry out a task, tend to anticipate potential barriers and take initiative to adopt new behaviours including health behaviours such as genetic screening [9]. Outcome expectancies refer to when an individual balances the pros and cons of the behavioural consequences [10]. Previous literature has focused on assessing positive outcome expectancies as these are the strongest predictors of intention [11]. Risk perception, which comprises perceived vulnerability and severity (from the Health Belief Model), is seen as more of a distal antecedent of outcome expectancies [12]. Risk perception alone is insufficient to enable a person to form an intention, however it is important in the formation of thoughts about consequences and competencies [9]. The Health Action Process Approach (HAPA) combines these cognitions in a causal structure to form an explanation of health behaviours and has previously demonstrated its value in explaining a number of health behaviours including breast and testicular self-examinations [9] [11] [13] [14].

Two additional belief-based factors that successfully predict intentions to undergo genetic screening are: attitude towards uncertainty [15] and anticipated regret [5]. Attitude towards uncertainty in the context of medical testing refers to the extent to which individuals desire to avoid health-related uncertainty [15]. Previous research has found uncertainty to be the main predictor of test-taking intentions, suggesting the desire to reduce uncertainty represents a potential motivating factor to undergo WGS [4] [6]. Anticipated regret following a health behaviour, such as genetic-testing, appears to be dependent on the test outcome (i.e., a positive result indicating an increased risk for a disease) and is predictive of lower genetic test-taking intentions [5]. The inclusion of these individual factors to the motivational stage of the HAPA model (see Figure 1) may potentially provide a more comprehensive understanding of intentions to undergo WGS, an area which has not been explored by previous psychosocial research.

This study aimed to: 1) determine whether particular disease characteristics; namely treatment and pene 


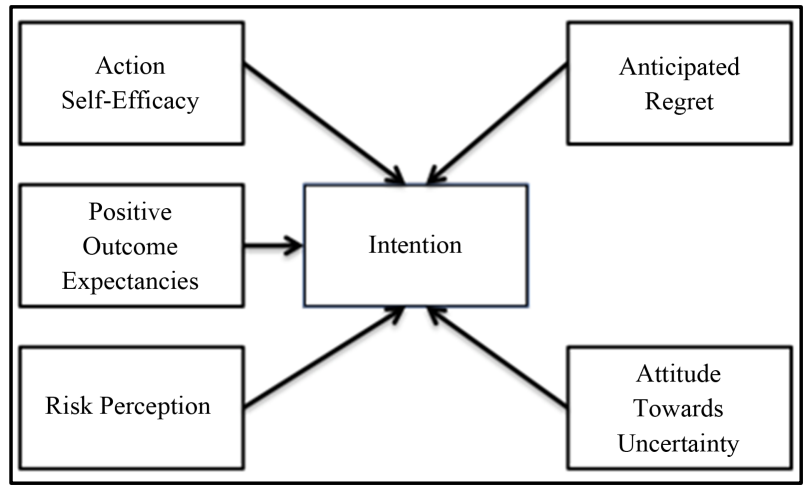

Figure 1. Health Action Process Approach factors and additional variables of interest influencing intention to undergo Whole Genome Sequencing.

trance, predict intentions to undergo WGS; 2) utilise the HAPA framework to investigate whether task self-efficacy, positive outcome expectancy and risk perception influence intentions to undertake WGS; and 3) investigate to what extent attitudes towards uncertainty and anticipated regret predict intentions to undergo WGS.

\section{Methods}

\subsection{Participants}

A convenience sample of 176 undergraduate psychology students was recruited via the University of Sydney database and received course credit for their participation. Ethics approval was granted by the university's Human Research Ethics Committee. Eligibility criteria included English proficiency and no previous experience of genetic testing.

\subsection{Materials and Measures}

All materials and measures were pilot tested using a sample of undergraduate students $(N=15)$ to assess understanding of hypothetical testing scenarios, identify any potential problems in questionnaire use and elicit any additional WGS-related beliefs. For all attitudinal measures (HAPA subscales, attitude towards uncertainty and anticipated regret scales) a total score was calculated (after reverse scoring relevant items), with higher scores indicating higher levels of the corresponding construct.

\subsubsection{WGS Information Pamphlet}

Prior to manipulation, participants read an introductory information pamphlet defining WGS and outlining the information it provides, its future availability to young people as well as its potential benefits and negative consequences in their lives.

\subsubsection{Hypothetical Disease Scenarios}

Four hypothetical scenarios were presented in the manipulation stage of the experiment, during which the participants had to imagine undergoing WGS and receiving their test results (see Appendix A for scenarios). The four scenarios systematically varied two disease characteristics: penetrance and treatment availability. Four fictitious diagnoses (Renibus disease, Iecur disease, Mesencephalon disorder and Prosencephalon disorder) were created for the scenarios to control for participants' prior knowledge and experiences of diseases, as potential confounding variables.

In the treatment available scenario participants received a positive test result for the invented Renibus or Iecur diseases and in the no treatment available scenario a predisposition to the fictitious Mesencephalon or Prosencephalon disorders, adapted from Roberts [8] and Shaw, Bassi [7] (see Table 1). Penetrance was operationalised using absolute risk values. Moderate penetrance was defined as a 50\% risk (50 out of 100 people will develop this disease in the future) and high penetrance indicated a 95\% chance (where 95 out of 100 people will develop this disease), based on previous research [5]. 
Table 1. Disease characteristics of each hypothetical disease scenario.

\begin{tabular}{cccc}
\hline & & & Availability of treatment \\
\cline { 2 - 4 } & & Treatment & No treatment \\
\hline Level of disease penetrance & $50 \%$ moderate & Scenario 1: Renibus disease & Scenario 3: Mesencephalon disorder \\
& $95 \%$ high & Scenario 2: Iecur disease & Scenario 4: Prosencephalon disorder \\
\hline
\end{tabular}

\subsubsection{HAPA Questionnaire}

The HAPA subscale items were developed by the authors based on past genetic research into attitudes and intentions in a similar population (for full list of items see Appendix B) [4]. All HAPA components, except intention, were assessed using multi-item measures with responses scored on seven point Likert-type scales: from very unlikely (1) to very likely (7) (task self-efficacy), from strongly disagree (1) to strongly agree (7) (positive outcome expectancies) and very low (1) to very high (7) (risk perception).

- Task self-efficacy: seven items, informed by the literature [13], assessed participants' beliefs about their capability of undergoing WGS when faced with specific challenges. Example question: "What is the likelihood you would undergo Whole Genome Screening if it involved undergoing a blood test?"

- Positive outcome expectancies: a 12-item subscale adapted from previous research [4] assessed participants' beliefs that the WGS outcomes will be favourable. Example question: "Whole Genome Screening would reduce distress and worry about future health."

- Risk perception: four items, informed by the literature [11] [16], assessed participants' beliefs about their vulnerability to particular illnesses. Example question: "I think my chances of having an increased risk for developing a type of cancer (e.g. breast, colorectal, ovarian, prostate or lung) are...”

- Intention: A single item measured participants' intentions to undergo WGS in the future after reading each hypothetical scenario, on a 10-point Likert type scale, ranging from very unlikely (0) to very likely (10).

The HAPA subscales displayed moderate $(\alpha=0.570$, risk perception) to high $(\alpha=0.800, \alpha=0.804$; task selfefficacy and positive outcome expectancy, respectively) internal reliability in the present study.

\subsubsection{Attitude towards Uncertainty}

The validated attitude towards uncertainty scale [15] assessed individual's tendency to avoid uncertainty in the specific context of medical testing. Eight items were rated on a five point Likert scale, ranging from strongly disagree (1) to strongly agree (5). The scale demonstrated high internal reliability in this sample $(\alpha=0.848)$.

\subsubsection{Anticipated Regret}

A purposed-designed six-item scale, developed from previous literature [5], measured an individual's anticipated regret following WGS dependent on different test results. Responses were scored on a five-item Likert scale from strongly disagree (1) to strongly agree (5). Anticipated regret scale demonstrated high reliability in this sample $(\alpha=0.745)$.

\subsubsection{Demographics Questionnaire}

Twelve items assessed participants': age, gender, ethnicity, biological children, personal and relatives’ genetic testing history and prior knowledge of WGS.

\subsubsection{Family and Personal History of Disease}

This 12-item scale, adapted from Fisher, Bonner, Biankin, and Juraskova [4] measured the number of first- and second-degree relatives affected by a series of common hereditary conditions. Each first-degree relative was assigned a value of 1 and each second-degree relative a value of 0.5 , these were summed together across all diseases, as per previous literature [4] [16] to compute a total family disease score. One item asked participants to indicate any personal history of chronic illness (yes; no; no answer).

\subsubsection{Knowledge of Genetics}

Two items, informed by Mackert, Rew, Bonevac, Champlin [17], assessed self-reported knowledge of human genetics on a five-point Likert scale from much lower than average (1) to much higher than average (5) and 
highest level of biology studied.

\subsection{Procedure}

The study procedure is summarised in Figure 2. After accessing the electronic survey on the Qualtrics survey system (www.qualtrics.com) via an email link and providing their consent, participants completed pre-manipulation measures and read each of the four hypothetical disease scenarios in a randomised order, reporting their intention to undergo WGS after each scenario. Participants then completed a series of post-manipulation measures including HAPA subscales, attitude towards uncertainty and anticipated regret scales, and were subsequently debriefed about the fictional nature of diseases.

\subsection{Data Analyses and Sample Size}

Statistical analyses were conducted using SPSS version 19 [18]. A within subjects $(2 \times 2)$ repeated measures ANCOVA was conducted to compare the effects of treatment availability (available, not available)and disease penetrance (50\%, 95\%)and their interaction on intention to undergo WGS, controlling for family history of disease. A series of Pearson's correlations were used to establish bivariate relationships between intention to undergo WGS, each of the HAPA components, attitude towards uncertainty and anticipated regret. A hierarchical multiple regression (method: enter) then tested the predictors of intention to undergo WGS with: family history of disease in block one, HAPA variables in block 2 and attitude towards uncertainty and anticipated regret in block 3. For all analyses, $p<0.05$ was considered statistically significant. Based on Fisher, Bonner, Biankin, and Juraskova [4] a medium effect size of 0.15 was assumed. To detect the medium effect size for 0.95 power and a 0.05 significance level with 6 predictors, a minimum sample of 107 participants was required [19].

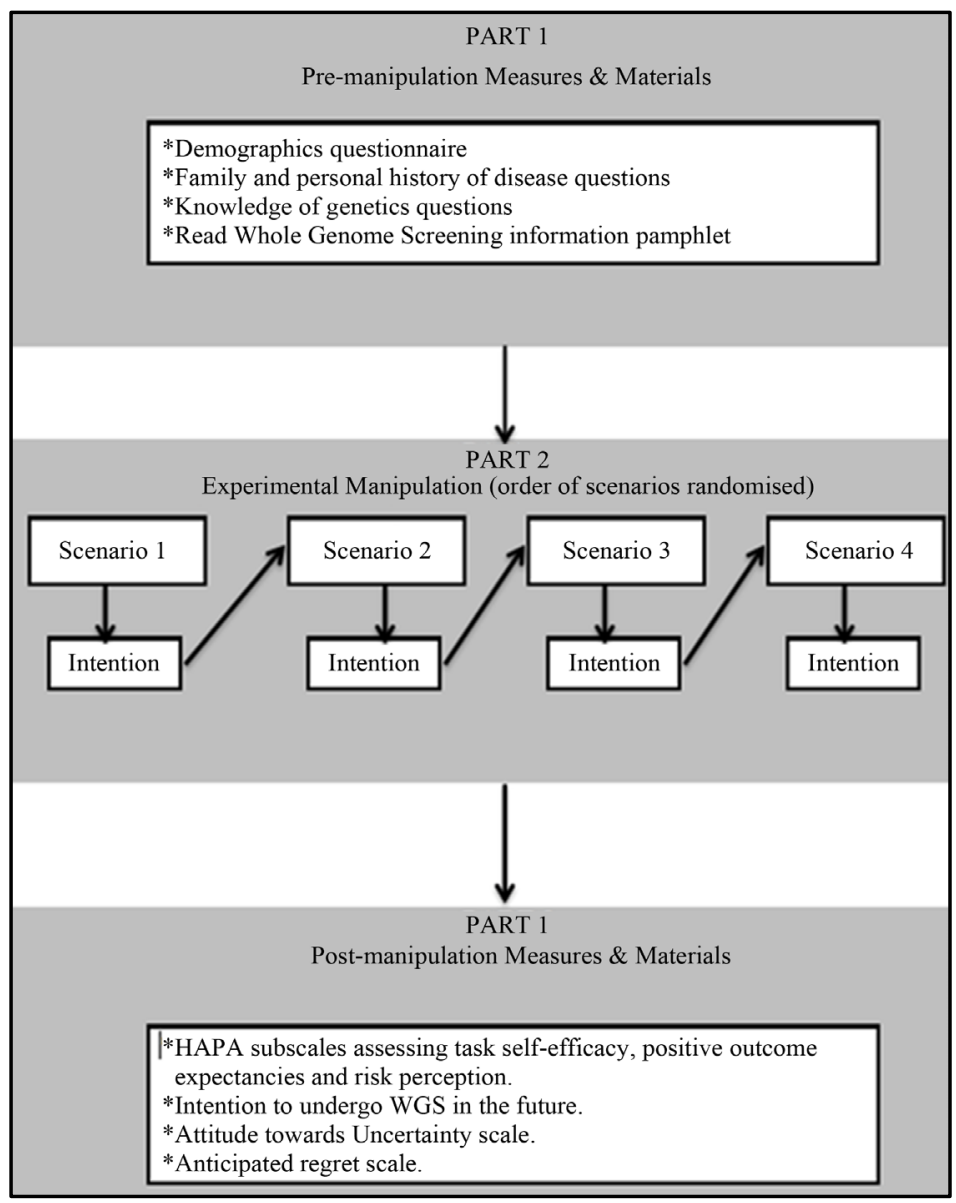

Figure 2. An illustrative diagram of the study procedure $(N=164)$. 


\section{Results}

\subsection{Sample}

Of 176 undergraduate university students recruited for the study, 8 participants did not to complete the study before the deadline, corresponding to a response rate of 95.4\%. Four participants who reported previously undergoing genetic testing were excluded from analyses as it could not be established if their responses were hypothetical or based on their previous experience. Therefore, the final sample comprised of 164 participants.

\subsubsection{Demographic Characteristics}

The mean age of participants was 19.7 years ( $S D=3.97$; range $17-46$ ). The majority of the sample were female (67\%), identified as Australian (65\%) and were single (68\%). Two participants reported having biological children and $14 \%$ of the sample reported that at least one of their relatives had undergone genetic testing. Over half (57\%) of participants indicated having prior knowledge of WGS, most commonly from school or university studies (39\%).

\subsubsection{Personal and Family History of Disease}

Twenty-five (15\%) participants reported suffering from a chronic illness at some stage in their life; the most common being depression $(n=9)$. Almost half the sample (44\%) reported having at least one first-degree relative affected by disease and the majority (87\%) reported at least one second-degree relative affected by disease.

\subsubsection{Knowledge of Biology and Genetics}

Almost half the sample (45\%) reported first-year university to be their highest level of biology study and rated their knowledge of genetics to be average (47\%). As the majority of participants self-reported average or belowaverage knowledge of human genetics (70\%), it was not deemed necessary to control for this variable in the statistical analysis.

\subsection{Post-Manipulation Measures}

Intention to undergo WGS testing was highest when disease penetrance was high and treatment options were available, compared to when disease penetrance was moderate and there were a lack of treatment options (see Table 2 for means and standard deviations). On average, participants reported moderate levels of task self-efficacy, positive outcome expectancy and risk perception as well as attitude towards uncertainty and anticipated regret.

\subsection{Impact of Disease Characteristics on WGS Intention}

A two-way repeated-measures ANCOVA revealed a significant main effect for treatment availability, controlling for family history of disease $(F(1,162)=113.951, p<0.001)$, with a large effect size (partial $\left.\eta^{2}=0.413\right)$. Participants reported significantly higher intention to undergo WGS in response to hypothetical scenarios where the disease was treatable compared non-treatable disease scenarios.

The two-way repeated-measures ANCOVA also revealed a significant main effect for penetrance, controlling for family history of disease $(F(1,162)=5.512, p=0.020)$, with a small effect size (partial $\eta^{2}=0.033$ ). Intention to undergo WGS was significantly higher when participants read hypothetical scenarios identifying a disease with high penetrance as opposed to moderate disease penetrance.

A significant interaction effect was found between treatment and penetrance, controlling for family history of disease $(F(1,162)=17.128, p<0.001)$. As illustrated in Table 3, the effect of available treatment compared to no available treatment on intention to undergo WGS was greater when disease penetrance was high than when it was moderate.

\subsection{Predictors of Intention to Undergo WGS}

To determine the predictors of WGS intention, independent factors that correlated significantly with intention were included in a hierarchical multiple regression. The overall regression was statistically significant $(F(4,139)$ $=62.339, p<0.001$ ) and the HAPA model explained $64.2 \%$ of the variance in WGS intention, combined with 
Table 2. Mean (standard deviation) intention to undergo WGS in each hypothetical scenario.

\begin{tabular}{cccc}
\hline & \multicolumn{3}{c}{ Availability of treatment } \\
\cline { 2 - 4 } & & Treatment & No treatment \\
\hline $\begin{array}{c}\text { Level of disease } \\
\text { penetrance }\end{array}$ & $50 \%$ moderate & $7.18(2.61)$ & $5.33(2.80)$ \\
& $95 \%$ high & $7.81(2.48)$ & $5.31(3.14)$ \\
\hline
\end{tabular}

Table 3. Hierarchical Multiple Regression analyses predicting intention to undergo WGS screening.

\begin{tabular}{|c|c|c|c|c|c|c|c|c|}
\hline Model & Predictor variables & B & SEB & $\beta$ & $t$ & $R^{2}$ & $\Delta R^{2}$ & $\Delta F$ \\
\hline \multirow[t]{2}{*}{1} & Constant & 5.561 & 0.329 & & 16.896 & 0.001 & 0.001 & 0.213 \\
\hline & Family history of disease & 0.059 & 0.128 & 0.039 & 0.461 & & & \\
\hline \multirow[t]{2}{*}{2} & Constant & -5.115 & 0.904 & & -5.656 & 0.642 & 0.641 & 82.924 \\
\hline & Family history of disease & -0.061 & 0.083 & -0.040 & -0.735 & & & \\
\hline HAPA & Task self-efficacy & 0.176 & 0.019 & 0.562 & $9.161^{b}$ & & & \\
\hline \multirow[t]{2}{*}{ Variables } & Positive outcome expectancy & 0.090 & 0.017 & 0.321 & $5.214^{\mathrm{b}}$ & & & \\
\hline & Risk perception & 0.062 & 0.034 & 0.101 & 1.841 & & & \\
\hline \multirow[t]{2}{*}{3} & Constant & -4.661 & 1.347 & & -3.460 & 0.660 & 0.018 & 3.632 \\
\hline & Family history of disease & -0.043 & 0.082 & -0.028 & -0.524 & & & \\
\hline HAPA & Task self-efficacy & 0.152 & 0.022 & 0.486 & $7.063^{\mathrm{b}}$ & & & \\
\hline \multirow[t]{2}{*}{ Variables } & Positive outcome expectancy & 0.068 & 0.019 & 0.242 & $3.589^{b}$ & & & \\
\hline & Risk perception & 0.050 & 0.034 & 0.080 & 1.477 & & & \\
\hline \multirow[t]{2}{*}{ Additional variables } & Attitude towards uncertainty & 0.073 & 0.031 & 0.179 & $2.376^{\mathrm{a}}$ & & & \\
\hline & Anticipated regret & -0.013 & 0.038 & -0.024 & -0.355 & & & \\
\hline
\end{tabular}

Note: Dependent variable: Intention to undergo WGS; ${ }^{\mathrm{a}} p<0.05,{ }^{\mathrm{b}} \mathrm{p}<0.001$.

family history of disease (see Table 3). In the first step (model 1), the control variable, family history of disease, was entered, however it did not significantly predict intention $F(1,142)=0.213, p=0.645$. In the second step, all HAPA components (task self-efficacy, positive outcome expectancy and risk perception) were added to the model and accounted for an additional $64.1 \%$ of the variance in intention, $\Delta F(3,139)=82.924, p<0.001$. In the final step (Model 3) HAPA components again accounted for a unique proportion of variance in intention, with task self-efficacy $(\beta=0.486, t(137)=7.063, p<0.001)$ and positive outcome expectancy $(\beta=0.242, t(137)=$ $3.589, p<0.001$ ) independently predicting intention while controlling for other predictors. Attitude towards uncertainty and anticipated regret were added into the multiple regression in model 3 and together accounted for an additional $1.8 \%$ of the variance in intention, a statistically significant proportion over and above the variance explained by HAPA variables and family history of disease $(\Delta F(2,137)=3.632, p=0.029)$. After controlling for other predictors, attitude towards uncertainty significantly predicted intention to undergo WGS $(\beta=0.179$, $t(137)=2.376, p=0.019)$, however, anticipated regret did not.

\section{Discussion}

As one of the first psychosocial studies to explore which disease and individual-based factors predict intention to pursue Whole Genome Screening (WGS) this study has a number of novel findings. As anticipated, the manipulation of disease characteristics in hypothetical testing scenarios led to differences in intention to undertake Whole Genome Sequencing (WGS). The availability of treatment influenced intention to undertake WGS, with respondents indicating significantly higher intention to undergo WGS when disease treatment was available as 
opposed to when no treatment was available, consistent with people's desire to assert control over their lives [20]. Respondents also indicated greater intention to pursue WGS in scenarios where disease penetrance was high in comparison to when penetrance was moderate. Thus healthy young adults seem more willing to undergo WGS when the presence of a genetic mutation denotes a more certain probability that the carrier will develop the disease they have tested positive for, consistent with genetic-testing literature [6] [8]. Therefore, the current findings suggest a role for available treatment and high disease penetrance on influencing screening intentions may apply in the genomic screening context.

Additionally, availability of treatment and penetrance worked in conjunction to influence intention to undergo WGS. Specifically, available treatment options appear to be more important when there is a greater than moderate probability of developing a disease. This novel interaction has not been observed in the genetic-testing literature potentially due to methodological differences between studies. One similar previous study manipulated disease penetrance across three levels (33\%, 66\% moderate or 100\% chance of developing the disease) and included an additional disease factor: severity [7]. Overall, the results indicate treatability and disease penetrance are two distinct motivations that can also interact to influence intention to pursue WGS.

The HAPA model provided a useful theoretical framework for predicting people's intention to undertake WGS to learn their personal disease susceptibility. HAPA components explained 64\% of the variance in screening intention, which is a notably greater amount than in a similar previous study, which applied another established psychosocial model of health behaviour (Protection Motivation Theory) to WGS intentions and included an information manipulation (36\%) [4]. In regards to the predictive power of each component of the HAPA model, task self-efficacy and positive outcome expectancy both significantly predicted intention, whilst controlling for the other components. Task self-efficacy emerged as the strongest predictor of WGS screening intentions in line with previous research identifying this component as the strongest and most robust predictor of intention to exercise and undergo breast and testicular self-examination behaviours [11] [14].

Contrary to predictions, risk perception did not independently predict WGS intentions. However, its lack of predictive power has been observed in previous studies, which argue risk perception is a distal antecedent factor involved in the initial formation of thoughts that fails to contribute a direct influence on intention [21] [22]. Further, Wade, Shiloh, Woolford, Roberts, Alford, Marteau, Biesecker [23] observed that perceived risk did not predict intention to obtain a genetic test for eight various health conditions as people did not perceive their risk to be equal for each condition. In the current study the perceived risk scale demonstrated low internal reliability. However, as WGS is a multifactorial test, it indicates personal risk to a number of single and multi-gene disorders, which carry varying degrees of objective risk. Considering also potential discrepancies between medical stratifications of risk and lay people's perception of risk, then perceived risk proves a particularly difficult construct to measure in this context. Future research could create diseases categories and measure risk perceptions for each category rather than pooling all types of diseases together.

Avoidance of health-related uncertainty also emerged as a significant independent predictor of screening intention, consistent with the genetic and genomic test-taking literature [4] [24]. This is despite WGS results not being able to provide definitive or certain outcomes as to whether an individual will develop a disease or not, even if a disease is known to have high penetrance. For example, Wolff, Nordin, Brun, Berglund, Kvale [6] found intolerance of uncertainty increased intention to undergo WGS, regardless of whether test results revealed a certain versus uncertain outcome (100\% vs. 50\% chance of developing a disease, respectively). Although WGS results may allay some uncertainties in personal disease susceptibility, people who prefer health-related certainty are likely to pursue WGS regardless.

Contrary to Frost, Myers, Newman 5 and Sweeny, Legg [25], anticipated regret did not independently predict WGS screening intention. The current study's use of a multi-item measure of anticipated regret could have captured a more complete assessment of anticipated regret but reduced its ability to predict screening intention [4]. Alternatively as Joseph-Williams, Edwards, Elwyn [26] observed, beliefs about regret are often dependent on a specific outcome including receiving a positive test-result as opposed to a negative result. As WGS is an untargeted approach to genetic analysis, it is unlikely to produce one disease-specific outcome but multiple outcomes conveying increased risk (or not) to numerous single-gene and multi-gene disorders simultaneously [2]. Therefore, the current measure of anticipated regret may not be relevant in the context of WGS.

\subsection{Limitations}

One of the study's potential limitations is the current sample, which was comprised of undergraduate university 
students who are unlikely to develop a chronic disease in the near future. This may potentially limit the generalisability of findings to the general population. Further, highly educated individuals have been shown to have a better capacity to engage with and understand genomic information and make better use of screening services [27]. As a consequence, lower health literacy among the general population could act as a potential barrier to WGS intention and uptake, as well as the communication of information both pre and post-screening. However, the current study has highlighted factors influencing intention among one of the potential target populations for WGS screening in the future, young adults [17]. Additionally, as the current sample reported limited knowledge of genomics and the test itself, it was possible to minimise the impact of pre-existing beliefs on intention.

Purposed-designed hypothetical testing scenarios were used to neutralise the effect of preconceived ideas regarding specific diseases and their potential psychological impact, and to ensure that the decision-making process was influenced only by relevant disease factors under investigation. We are aware that hypothetical intention to undertake WGS may not be equivalent to actual test-uptake, as previous literature indicates a considerable discrepancy between high self-reported intentions and lower actual test uptake in genetic screening [23] [28]. As WGS is currently very expensive with limited availability, it was not feasible for the current study to measure test uptake. Thus, intention provided the best proxy for actual behaviour in this setting.

\subsection{Conclusions}

To the authors' knowledge this is one of the first psychosocial studies to explore the impact of disease characteristics on intention to undertake WGS. The findings suggest that, in this population of young and healthy adults, WGS intention is likely to be influenced by disease-based factors, namely whether treatment for a hereditary condition is available and the probability that a genetic marker will develop into disease. WGS screening providers and researchers need to take these disease characteristics into account when designing screening information materials, ensuring that they accommodate participant preferences for information, while still providing balanced information to ensure informed consent.

Considering, however, the vast amount of information and knowledge that is offered by WGS results, obtaining informed consent for this procedure may prove more complex than for single-gene tests. Future research should involve the development of decision-making resources, clearly explaining the WGS procedure, benefits, limitations and associated risks, which have proved beneficial for genetic testing for single-gene conditions previously [29] [30]. Given that, among young and healthy adults, particular individual factors such as task self-efficacy, positive outcome expectancy and attitude towards uncertainty are likely to motivate screening intentions, screening promotions in a clinical setting could increase intentions in eligible patients by targeting these factors. Moreover, the desire to reduce health-related uncertainty can be a potentially motivating factor in this setting. Thus, WGS providers must promote awareness that screening results may find a very small increased risk of disease with no clear course of action [31] to counteract misconceptions about the predictive power of WGS [32].

With the unprecedented speed at which WGS technologies are advancing and their increasing availability to the public, further psychosocial research is needed to determine which factors influence screening intention and actual uptake in clinical populations. Evidence from this research may be used to develop empirically-based psycho-educational materials for consumers, as well as inform the optimal design of screening provider protocols in this setting.

\section{Acknowledgements}

We acknowledge support from the University of Sydney and substantial support and feedback from our colleague Ilan Dar-Nimrod.

\section{References}

[1] De Cristofaro, E. (2012) Whole Genome Sequencing: Innovation Dream or Privacy Nightmare? CoRR, 1-9.

[2] Offit, K. (2011) Personalized Medicine: New Genomics, Old Lessons. Human Genetics, 130, 3-14. http://dx.doi.org/10.1007/s00439-011-1028-3

[3] Moorthie, S., Hall, A. and Wright, C.F. (2013) Informatics and Clinical Genome Sequencing: Opening the Black Box. Genetics in Medicine, 15, 165-171. http://dx.doi.org/10.1038/gim.2012.116

[4] Fisher, A., Bonner, C., Biankin, A.V. and Juraskova, I. (2012) Factors Influencing Intention to Undergo Whole Genome 
Screening in Future Healthcare: A Single-Blind Parallel-Group Randomised Trial. Preventive Medicine, 55, 514-520. http://dx.doi.org/10.1016/j.ypmed.2012.08.008

[5] Frost, S., Myers, L.B. and Newman, S.P. (2001) Genetic Screening for Alzheimer's Disease: What Factors Predict Intentions to Take a Test? Behavioral Medicine, 27, 101-109. http://dx.doi.org/10.1080/08964280109595776

[6] Wolff, K., Nordin, K., Brun, W., Berglund, G. and Kvale, G. (2011) Affective and Cognitive Attitudes, Uncertainty Avoidance and Intention to Obtain Genetic Testing: An Extension of the Theory of Planned Behaviour. Psychology \& Health, 26, 1143-1155. http://dx.doi.org/10.1080/08870441003763253

[7] Shaw, J.S., and Bassi, K.L. (2001) Lay Attitudes toward Genetic Testing for Susceptibility to Inherited Diseases. Journal of Health Psychology, 6, 405-423. http://dx.doi.org/10.1177/135910530100600404

[8] Roberts, J.S. (2000) Anticipating Response to Predictive Genetic Testing for Alzheimer's Disease: A Survey of FirstDegree Relatives. The Gerontologist, 40, 43-52. http://dx.doi.org/10.1093/geront/40.1.43

[9] Schwarzer, R. and Luszczynska, A. (2008) How to Overcome Health-Compromising Behaviors: The Health Action Process Approach. European Psychologist, 13, 141-151. http://dx.doi.org/10.1027/1016-9040.13.2.141

[10] Schwarzer, R. (2008) Modeling Health Behavior Change: How to Predict and Modify the Adoption and Maintenance of Health Behaviors. Applied Psychology, 57, 1-29.

[11] Scholz, U., Keller, R. and Perren, S. (2009) Predicting Behavioral Intentions and Physical Exercise: A Test of the Health Action Process Approach at the Intrapersonal Level. Health Psychology, 28, 702-708. http://dx.doi.org/10.1037/a0016088

[12] Bunn, J.Y., Bosompra, K., Ashikaga, T., Flynn, B.S. and Worden, J.K. (2002) Factors Influencing Intention to Obtain a Genetic Test for Colon Cancer Risk: A Population-Based Study. Preventive Medicine, 34, 567-577. http://dx.doi.org/10.1006/pmed.2002.1031

[13] Schwarzer, R. and Renner, B. (2000) Social-Cognitive Predictors of Health Behavior: Action Self-Efficacy and Coping Self-Efficacy. Health Psychology, 19, 487-495. http://dx.doi.org/10.1037/0278-6133.19.5.487

[14] Schwarzer, R. (1992) Self-Efficacy in the Adoption and Maintenance of Health Behaviours: Theoretical Approaches to a New Model. In: Schwarzer, R., Ed., Self-Efficacy: Thought Control of Action, Hemisphere Publishing, Washington DC, 217-242.

[15] Braithwaite, D., Sutton, S. and Steggles, N. (2002) Intention to Participate in Predictive Genetic Testing for Hereditary Cancer: The Role of Attitude toward Uncertainty. Psychology \& Health, 17, 761-772. http://dx.doi.org/10.1080/0887044021000054764

[16] Cameron, L.D. and Diefenbach, M.A. (2001) Responses to Information about Psychosocial Consequences of Genetic Testing for Breast Cancer Susceptibility: Influences of Cancer Worry and Risk Perceptions. Journal of Health Psychology, 6, 47-59. http://dx.doi.org/10.1177/135910530100600104

[17] Mackert, M., Rew, L., Bonevac, D. and Champlin, S. (2012) Older Adolescents’ Perceptions and Intentions Regarding Do-It-Yourself Genetic Assessment Services. Journal for Specialists in Pediatric Nursing, 17, 159-167.

[18] IBM Corporation (2010) Statistical Package for the Social Sciences (SPSS) for Macintosh [Computer Program]. Version 19.0. Armonk.

[19] Tabachnick, B.G. and Fidell, L.S. (2007) Using Multivariate Statistics. 5th Edition, Pearson Education Limited, Upper Saddle River.

[20] Lerman, C., Croyle, R.T., Tercyak, K.P. and Hamann, H. (2002) Genetic Testing: Psychological Aspects and Implications. Journal of Consulting and Clinical Psychology, 70, 784-797. http://dx.doi.org/10.1037/0022-006X.70.3.784

[21] Cameron, L.D. and Reeve, J. (2006) Risk Perceptions, Worry, and Attitudes about Genetic Testing for Breast Cancer Susceptibility. Psychology \& Health, 21, 211-230. http://dx.doi.org/10.1080/14768320500230318

[22] Schwarzer, R., Lippke, S. and Luszczynska, A. (2011) Mechanisms of Health Behavior Change in Persons with Chronic Illness or Disability: The Health Action Process Approach (HAPA). Rehabilitation Psychology, 56, 161-170. http://dx.doi.org/10.1037/a0024509

[23] Wade, C.H., Shiloh, S., Woolford, S.W., Roberts, J.S., Alford, S.H., Marteau, T.M. and Biesecker, B.B. (2012) Modelling Decisions to Undergo Genetic Testing for Susceptibility to Common Health Conditions: An Ancillary Study of the Multiplex Initiative. Psychology \& Health, 27, 430-444. http://dx.doi.org/10.1080/08870446.2011.586699

[24] Croyle, R.T., Dutson, D.S., Tran, V.T. and Sun, Y.C. (1995) Need for Certainty and Interest in Genetic Testing. Women's Health: Research on Gender, Behavior and Policy, 1, 329-339.

[25] Sweeny, K. and Legg, A.M. (2011) Predictors of Interest in Direct-to-Consumer Genetic Testing. Psychology \& Health, 26, 1259-1272. http://dx.doi.org/10.1080/08870446.2010.514607

[26] Joseph-Williams, N., Edwards, A. and Elwyn, G. (2011) The Importance and Complexity of Regret in the Measurement of “Good” Decisions: A Systematic Review and a Content Analysis of Existing Assessment Instruments. Health 
Expectations, 14, 59-83. http://dx.doi.org/10.1111/j.1369-7625.2010.00621.x

[27] McBride, C.M., Koehly, L.M., Sanderson, S.C. and Kaphingst, K.A. (2010) The Behavioral Response to Personalized Genetic Information: Will Genetic Risk Profiles Motivate Individuals and Families to Choose More Healthful Behaviors? Annual Review of Public Health, 31, 89-103. http://dx.doi.org/10.1146/annurev.publhealth.012809.103532

[28] Binedell, J. and Soldan, J.R. (1997) Nonparticipation in Huntington's Disease Predictive Testing: Reasons for Caution in Interpreting Findings. Journal of Genetic Counseling, 6, 419-432. http://dx.doi.org/10.1023/A:1025601010891

[29] Grosse, S.D., McBride, C.M., Evans, J.P. and Khoury, M.J. (2009) Personal Utility and Genomic Information: Look before You Leap. Genetics in Medicine, 11, 575-576. http://dx.doi.org/10.1097/GIM.0b013e3181af0a80

[30] Iredale, R., Rapport, F., Sivell, S., Jones, W., Edwards, A., Gray, J. and Elwyn, G. (2008) Exploring the Requirements for a Decision Aid on Familial Breast Cancer in the UK Context: A Qualitative Study with Patients Referred to a Cancer Genetics Service. Journal of Evaluation in Clinical Practice, 14, 110-115. http://dx.doi.org/10.1111/j.1365-2753.2007.00811.x

[31] Kuehn, B.M. (2008) Risks and Benefits of Direct-to-Consumer Genetic Testing Remain Unclear. Journal of the American Medical Association, 300, 1503-1505. http://dx.doi.org/10.1001/jama.300.13.1503

[32] Leighton, J.W., Valverde, K. and Bernhardt, B.A. (2011) The General Public's Understanding and Perception of Directto-Consumer Genetic Test Results. Public Health Genomics, 15, 11-21. http://dx.doi.org/10.1159/000327159 


\section{Appendix A}

\section{Scenario 1-Renibus Disease}

Imagine you have just undergone whole genome sequencing. The test results identify you have a genetic predisposition to develop Renibus disease in the future. Renibus disease is progressive leakage of protein from the glomerulus in the kidneyleading to kidney failure. When the kidneys stop working most people develop conditions that affect their blood, bones, nerves and skins and have an increased risk of cardiovascular disease. Your doctor informs you there is effective treatment available to cure the Renibus disease if it develops. The test finds you have a 50\% chance of this genetic mutation developing into Renibus disease; meaning 50 in every 100 people with this result will develop Renibus disease in their lifetime.

\section{Scenario 2-Iecur Disease}

Imagine you have just undergone whole genome sequencing. The test results identify you have a genetic predisposition to develop Iecur disease in the future. Iecur disease is immunological destruction to the liver cells leading to liver failure. Liver failure can cause many health consequences including excessive bleeding and increase pressure in the brain. Your doctor informs you there is effective treatment available to cure Iecur disease if it develops. The test finds you have a 95\% chance of this genetic mutation developing into Iecur disease; meaning 95 in every 100 people with this result will develop Iecur disease in their lifetime.

\section{Scenario 3-Mesencephalon Disorder}

Imagine you have just undergone whole genome sequencing. The test results identify you have a genetic predisposition to develop Mesencephalon disorder. Mesencephalon disorder is a neurological condition that leads to progressive deterioration of sensory functions including vision and hearing. Your doctor informs you there is no treatment available to cure Mesencephalon disorder if it develops. The test finds you have a 50\% chance of this genetic mutation developing into Mesencephalon disorder; meaning 50 in every 100 people with this result will develop Mesencephalon disorder in their lifetime.

\section{Scenario 4-Prosencephalon Disorder (Forebrain/Frontal Lobe)}

Imagine you have just undergone whole genome sequencing. The test results identify you have a genetic predisposition to develop Prosencephalon disorder. Prosencephalon disorder is a neurological condition that leads to progressive deterioration in the function of memory, planning, motivation and aspects of emotional function. Your doctor informs you there is no treatment available to cure the Prosencephalon disorder if it develops. The test finds you have a 95\% chance of this genetic mutation developing into Prosencephalon disorder; meaning 95 in every 100 people with this result will develop Prosencephalon disorder in their lifetime.

\section{Appendix B}

\section{Health Action Process Approach Scale}

\section{Risk Perception Subscale}

This subscale asks you to rate the likelihood of you having an increased risk for different types of disease. For each of the statements below, please indicate the best/most appropriate response for you.

On a scale of 1 (very low) to 7 (very high), I think my chances of having an increased risk for developing.

1) A common disease (for example diabetes type 2 or heart disease) caused by genes, my behaviour and the environment.

2) A type of cancer (breast, colorectal, ovarian, prostate, lung).

3) A psychiatric condition (bipolar disorder, schizophrenia disorder, major depression).

4) A neurodegenerative disease (Alzheimer's disease, Parkinson's disease or Huntington's disease).

\section{Positive Outcome Expectancy Subscale}

This section will look at your beliefs about the benefits and advantages of undergoing whole genome sequencing. Please indicate the extent to which you agree with the following statements, on a scale of 1 (strongly disagree) 
to 7 (strongly agree) Whole genome screening would...

1) Enable early detection of and treatment for diseases for which I may have an increased risk of developing.

2) Enable me to come to terms with and prepare for the possibility of developing a disease.

3) Enable me to discover my personal susceptibility to diseases.

4) Increase my awareness and understanding of the disease I am at risk of developing.

5) Reduce distress and worry about my future health.

6) Help family planning by telling me about my future health and disease risk.

7) Inform my family of familial disease risk.

8) Enable me to get counselling and support for my disease risk and future health.

9) ${ }^{*}$ Give me information that is currently not useful for managing my health.

10) ${ }^{*}$ Give me information that is inaccurate or contains errors.

11) ${ }^{*}$ Give me information that scientists cannot currently interpret or explain what it means.

Task Self-Efficacy Subscale

Various barriers make it hard to undergo whole genome sequencing.

On a scale of 1 (very unlikely) to 7 (very likely) what is the likelihood you would undergo Whole Genome Sequencing if you had to...

1) Undergo a blood test.

2) Pay $\$ 1000$ for the test.

3) Undergo the test against your family’s wishes.

4) Communicate the results to your family.

5) If results may put you at risk of discrimination from insurance companies and employers.

\section{Intention Subscale}

Now you are aware of multiple very different possible outcomes of whole genome sequencing, please indicate your likelihood of undergoing whole genome sequencing in the future when the test becomes available.

Please click at the point on the line from 0 - 10, which corresponds to your likelihood of undergoing whole genome sequencing in the future. 
Scientific Research Publishing (SCIRP) is one of the largest Open Access journal publishers. It is currently publishing more than 200 open access, online, peer-reviewed journals covering a wide range of academic disciplines. SCIRP serves the worldwide academic communities and contributes to the progress and application of science with its publication.

Other selected journals from SCIRP are listed as below. Submit your manuscript to us via either submit@scirp.org or Online Submission Portal.
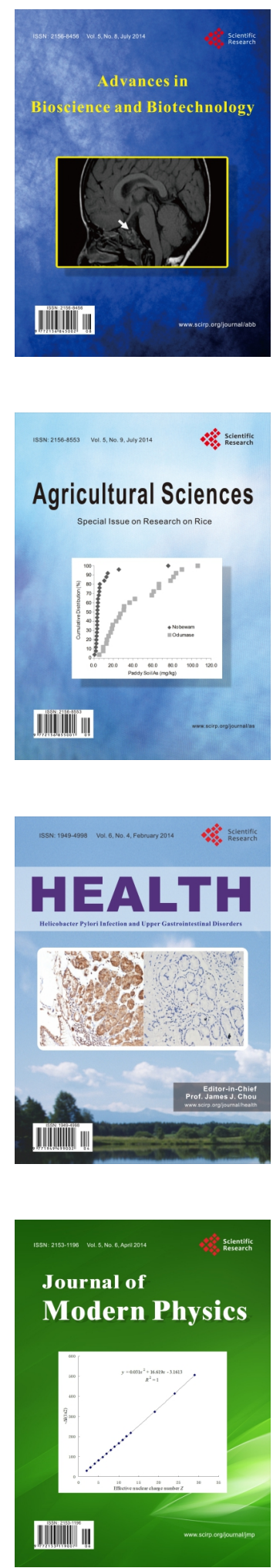
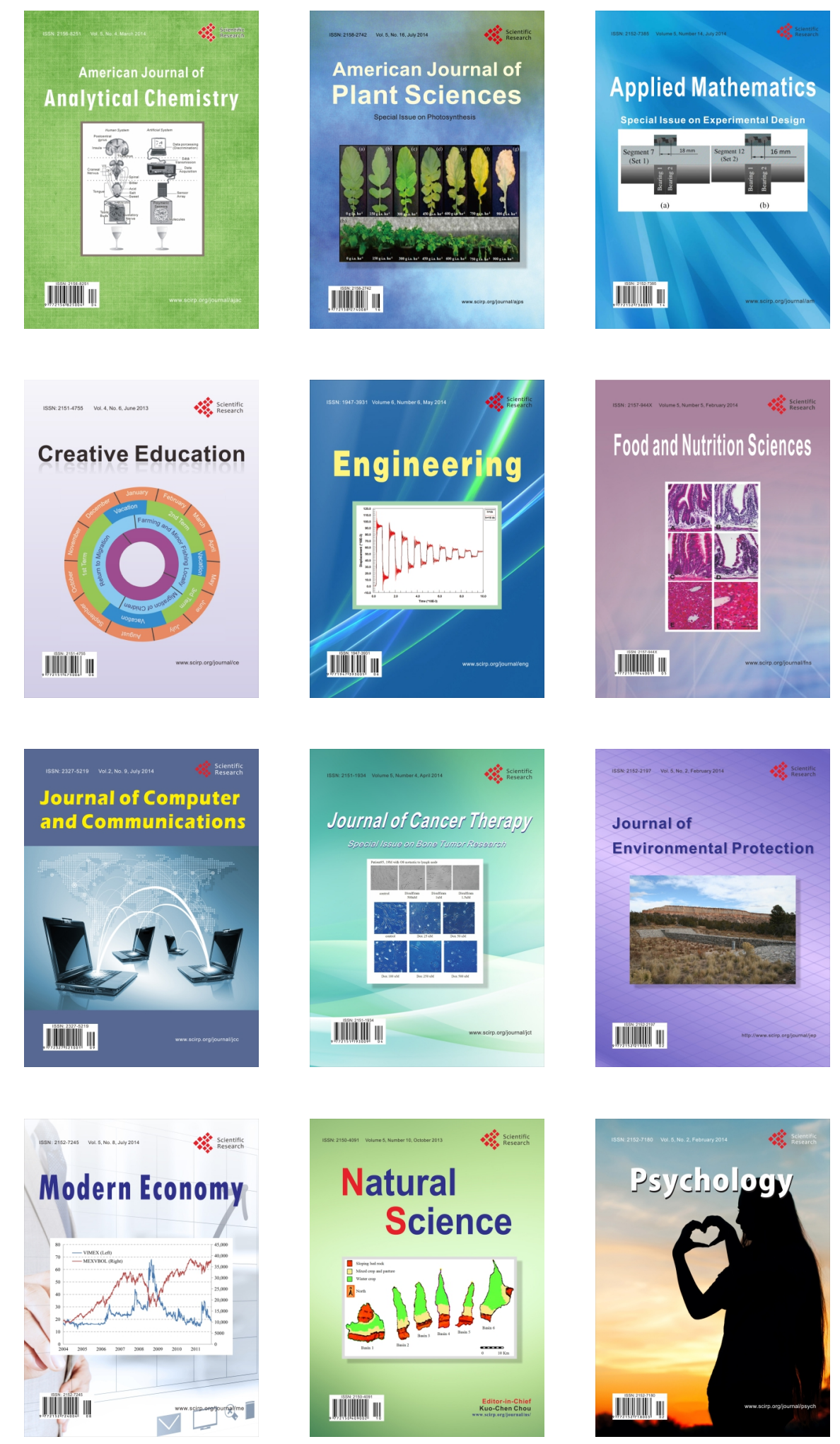